\title{
CORRECTION
}

View Article Online

View Journal I View Issue

\section{D) Check for updates Correction: Dynamical self-assembly of dipolar active Brownian particles in two dimensions}

Cite this: Soft Matter, 2020,

16,6443

Guo-Jun Liao, ${ }^{\star a}$ Carol K. Hall ${ }^{b}$ and Sabine H. L. Klapp ${ }^{\star a}$

Correction for 'Dynamical self-assembly of dipolar active Brownian particles in two dimensions' by

DOI: $10.1039 / \mathrm{d} 0$ sm90129f

Guo-Jun Liao et al., Soft Matter, 2020, 16, 2208-2223, DOI: 10.1039/C9SM01539F.

rsc.li/soft-matter-journal

The authors regret the errors in eqn (22) and (26) in the original manuscript. The correct versions of these equations are as shown below.

Eqn (22):

$$
\begin{aligned}
\boldsymbol{F}_{i, \mathrm{dd}}^{R}= & -\sum_{i \neq j}\left[\left(\left(\boldsymbol{\mu}_{i} \cdot \boldsymbol{\mu}_{j}\right) \boldsymbol{r}_{i j}+\boldsymbol{\mu}_{i}\left(\boldsymbol{\mu}_{j} \cdot \boldsymbol{r}_{i j}\right)+\boldsymbol{\mu}_{j}\left(\boldsymbol{\mu}_{i} \cdot \boldsymbol{r}_{i j}\right)\right) C\left(r_{i j}, \alpha\right)\right. \\
& \left.-\left(\boldsymbol{\mu}_{i} \cdot \boldsymbol{r}_{i j}\right)\left(\boldsymbol{\mu}_{j} \cdot \boldsymbol{r}_{i j}\right) \boldsymbol{r}_{i j} D\left(r_{i j}, \alpha\right)\right],
\end{aligned}
$$

where the distance vector is defined as $\boldsymbol{r}_{i j}=\boldsymbol{r}_{j}-\boldsymbol{r}_{i}$.

Eqn (26):

$$
\boldsymbol{T}_{i}^{R}=-\sum_{j \neq i}\left[\left(\boldsymbol{\mu}_{i} \times \boldsymbol{\mu}_{j}\right) B\left(r_{i j}, \alpha\right)-\left(\boldsymbol{\mu}_{i} \times \boldsymbol{r}_{i j}\right)\left(\boldsymbol{\mu}_{j} \cdot \boldsymbol{r}_{i j}\right) C\left(r_{i j}, \alpha\right)\right]
$$

The Royal Society of Chemistry apologises for these errors and any consequent inconvenience to authors and readers.

\footnotetext{
${ }^{a}$ Institut für Theoretische Physik, Technische Universität Berlin, Hardenbergstr. 36, D-10623 Berlin, Germany. E-mail: guo-jun.liao@campus.tu-berlin.de, klapp@physik.tu-berlin.de

${ }^{b}$ Department of Chemical \& Biomolecular Engineering, North Carolina State University, Raleigh, NC 27695, USA
} 